\title{
Atención y raciocinio visoespacial en jugadores de voleibol de alto rendimiento
}

\author{
Attention and visuospatial reasoning in high-performance volleyball players
}

Atenção e raciocínio visuoespacial em jogadores de voleibol de alto rendimento

\author{
Antonio Luiz Prado Serenini ${ }^{1}$, ORCID 0000-0002-7436-3440 \\ Fabián J. M. Rueda ${ }^{2}$, ORCID 0000-0001-5173-0802 \\ María Perla Moreno ${ }^{3}$, ORCID 0000-0003-0405-2409 \\ 12 Universidade São Francisco. Brasil \\ ${ }^{3}$ Universidad de Extremadura. España
}

\begin{abstract}
Resumen: Este estudio tuvo como objetivo analizar las relaciones de la atención y el raciocínio visoespacial con el rendimiento deportivo, en el contexto de voleibol. Participaron 159 jugadores de voleibol de alto nivel, de ambos sexos, con edad promedio de 21.60 años $(D E=4.11)$. Los instrumentos utilizados fueron la Bateria Psicológica de Evaluación de la Atención (BPA), Escala de Atención Selectiva Visual (EASV) y Test de los Cubos. El rendimiento deportivo fue medido utilizando el software Data Volley. Los resultados indican que los jugadores obtuvieron medias superiores a los patrones normativos de los manuales, en todas las pruebas, y sólo se encontraron correlaciones significativas entre los test y el rendimiento deportivo en el test BPA en la categoría juvenil, y en el Test de los Cubos en la categoría infanto. Los resultados de los test (BPA, EASV y Test de los Cubos) de forma general, y en categorías deportivas, no presentaron correlaciones significativas con el rendimiento deportivo.
\end{abstract}

Palabras clave: atención, evaluación, raciocínio visoespacial, rendimiento deportivo

Abstract: The present study aims at analyzing the relationship of attention and visuospatial reasoning to sports performance in the volleyball context. Participants were 159 high-level volleyball athletes of both sexes, with an average age of 21.60 years $(S D=4.11)$. Participants completed the Psychological Battery of Attention Assessment (BPA), the Visual Selective Attention Scale and the Cubes test, which were applied collectively. The sports performance was assessed using the DataVolley program. Results indicated that athletes scored above average on all tests when compared to available normative data. BPA scores and sports performance were correlated among adolescents, and Cubes test and sports performance were correlated among children. Overall and across sport categories, test scores (BPA, EASV and Cubes) did not associate with sports performance.

Keywords: attention, assessment, visuospatial reasoning, sports performance 
Resumo: Este estudo teve como objetivo analizar as relações entre atenção e raciocínio visuoespacial com o rendimento esportivo no contexto do voleibol. Participaram 159 jogadores de voleibol de alto rendimento, ambos os sexos, com idade média de 21,60 anos $(D P=4,11)$. Os instrumentos utilizados foram a Bateria Psicológica para Avaliação da Atenção (BPA), Escala de Atenção Selectiva Visual (EASV) y Teste dos Cubos. O rendimento esportivo foi mensurado por meio do software Data Volley. Os resultados indicam que os jogadores tiveram, em todas as provas, medias superiores às medias normativas dos manuais dos testes, y foram verificadas correlações significativas apenas entre os testes e o rendimento esportivo na BPA na categoria juvenil, e no Teste dos Cubos na categoria infantil. Os resultados dos testes (BPA, EASV e Teste dos Cubos) de forma geral, e em categorias esportivas não apresentaram correlações significativas com o rendimento esportivo.

Palavras-chave: atenção, avaliação, raciocínio visuoespacial, rendimento esportivo

Recibido: $26 / 11 / 2019$

Aceptado: 20/10/2020

Cómo citar:

Serenini, A. L. P., Rueda, F. J. M. \& Moreno, M. P. (2021). Atención y raciocinio visoespacial en jugadores de voleibol de alto rendimiento. Ciencias Psicológicas, 15(1), e-2348. doi: ttps://doi.org/10.22235/cp.v15i1.2348

Correspondencia:Fabián J. M. Rueda, Universidade São Francisco, Brasil. E-mail: marinfabian@ gmail.com

En el deporte de alto rendimiento, los resultados están directamente relacionados con el dominio eficiente de las capacidades físicas, técnicas, tácticas y psicológicas de los deportistas (Janelle \& Hillman, 2003), pudiendo adquirirse este dominio por medio de años de entrenamiento en varias fases de la formación deportiva. En las últimas décadas, los estudios han intentado analizar la influencia y/o relación de las capacidades cognitivas con el deporte, y principalmente sus relaciones con el rendimiento, de forma general (Castro, Praça, De Conti, Pedrosa \& Greco, 2016; Conejero, Serenini, Fernández-Echeverria, Collado-Mateo, \& Moreno, 2020; Orlick, 2007; Swann, Moran \& Piggot, 2015).

En los juegos deportivos colectivos, como voleibol, fútbol, balonmano, baloncesto, por ejemplo, la influencia de las capacidades cognitivas es muy importante, ya que los jugadores necesitan, en todo momento, percibir y analizar las posiciones de sus compañeros y de sus adversarios, así como el movimiento del balón dentro del terreno de juego, para tomar decisiones (Cárdenas, Perales \& Alarcón, 2015; Conejero, Serenini, Gonzáles-Silva \& Moreno, 2020; Suárez-Cadenas, Courel-Ibáñes \& Cárdenas-Vélez, 2017; Matias \& Greco, 2010; McAuliffe, 2004; Thomas, 1994). La habilidad para atender a esas demandas durante el juego diferencia a los deportistas en cuanto a sus realizaciones tácticas y a las respuestas que dan en las distintas situaciones. De acuerdo con Costa, Garganta, Fonseca y Botelho (2002) y Wulf y Prinz (2001), entre las capacidades cognitivas, la atención y el raciocínio visoespacial ejercen una gran influencia en el rendimiento en los juegos deportivos colectivos, en la medida en que las acciones son siempre variadas y requieren altos niveles de análisis de 
la información, para que ocurran toma de decisiones eficientes. Por otro lado, estudios también han buscado observar si la actividad física ejerce influencia sobre las funciones ejecutivas. En este sentido, por medio de una revisión sistemática, Medina-Cascales, Alarcón-López, Castillo-Díaz y Cárdenas-Vélez (2019), consideraron factores mediadores del efecto del ejercicio físico en las funciones ejecutivas desde el punto de vista cuantitativo y cualitativo. As estudiar niños y jóvenes, los autores no encontraron evidencias suficientemente sólidas sobre esa relación. Destacan la existencia de pocos estudios con rigor metodológico que permitan llegar a esa relación, una vez que los aspectos cualitativos de la actividad física no son considerados la mayor parte de las veces. De esta forma, destacan la necesidad de incluir aspectos como la manipulación de la carga mental durante la actividad física. Otros autores, como García, Garcés y Jara (2005) también destacan que en el ámbito del deporte los estudios son escasos al referirse a los procesos atencionales.

La atención puede ser entendida como la capacidad de procesamiento de una cantidad limitada de información en relación con una cantidad bastante grande disponible, por los órganos de los sentidos, de memoria almacenada y de otros aspectos cognitivos (Sternberg, 2000). En el ámbito deportivo, la atención representa la capacidad de controlar la percepción y los procesos de pensamiento de forma concentrada en los estímulos apropiados durante los entrenamientos y las competiciones, desconsiderando los estímulos perturbadores para conseguir una actuación eficiente y eficaz (Abernethy, 2001; Orlick, 2007).

Por su parte, el raciocínio visoespacial es definido como la capacidad para codificar, recordar, transformar y combinar estímulos mentalmente, modificando las imágenes visuales (Carroll, 1993), y está relacionado a la habilidad de usar las capacidades de imaginación mental para solucionar problemas, implicando los procesos de generar, percibir, analizar, almacenar, recordar, manipular y transformar representaciones o patrones visuales (McGrew, 2009; Schneider \& McGrew, 2012). El dominio de esa capacidad ejerce gran influencia en la realización de acciones deportivas, de forma general, en la medida en que el deportista procesa información visoespacial con relación a las personas, implementos y al espacio donde ocurren las acciones (Memmert, Simons \& Grimme, 2009).

Los estudios que comparan el rendimiento de deportistas expertos y noveles han tratado de analizar los niveles de atención y de raciocínio visoespacial y su relación con el rendimiento deportivo y con otras variables. Uno de los factores analizados es la relación del tipo de foco utilizado por los deportistas en diferentes fases de rendimiento deportivo, observándose que, mientras los noveles utilizan más el foco interno, los deportistas con más experiencia utilizan el foco externo (Beilock, Carr, MacMahon \& Starkes, 2002; Castaneda \& Gray, 2007; Miçooğulları \& Kirazci, 2012). Los deportistas noveles, al no dominar las acciones de forma automática, tienen que mantener el foco interno para coordinar de forma eficiente los movimientos. Sin embargo, los deportistas expertos, como dominan las acciones motoras, orientan el foco de atención a los factores externos y a los estímulos del entorno (Cárdenas et al., 2015; Wulf \& Su, 2007). Otras investigaciones han analizado los efectos de una segunda tarea simultánea y su interferencia en el rendimiento y en el aprendizaje de deportistas noveles y expertos (Emanuel, Jarus \& Bart, 2008; Gray, 2004; Sindik, Botica \& Fiškuš, 2015; Wulf, Shea \& Park, 2001), indicando que los deportistas noveles sufren más influencia de una segunda tarea, que los deportistas con más experiencia, en función del menor dominio de los procesos atencionales, de memoria a largo plazo y de velocidad de raciocínio.

En lo que respecta a los expertos, desarrollan mecanismos más efectivos de control de los procesos de atención y de raciocínio visoespacial, encontrándose las principales diferencias con los noveles en función de su capacidad de percepción, de análisis y del tiempo de respuesta a los estímulos correctos, y también de la capacidad de discriminar los estímulos irrelevantes y falsos de manera más eficiente que los noveles (Moran, 2012; Ripoll, 1991; 
Sánchez-López, Fernández, Silva-Pereyra, Martínez-Mesa \& Moreno-Aguirre, 2014). Los deportistas expertos, a su vez, detectan mejor la información relevante de su deporte, y desarrollan estrategias más elaboradas de búsqueda visual. Su comportamiento visual realiza menos fijaciones, con mayor duración, selecionando de forma más eficiente la información visual disponible (Afonso, Garganta, McRobert, Williams \& Mesquita, 2012; Dick, Button \& Davids, 2010). Esas características les permiten obtener una mayor información sobre los elementos más relevantes y significativos para la toma de decisiones en situación de juego, en función de una búsqueda especializada

La cuestión entre expertos y noveles fue ampliamente investigada por Medina (2017). Al citar a Williams, Davids y Williams (1999), el autor destaca que 'para alcanzar el éxito deportivo, los jugadores de élite ponen en acción sus destrezas y habilidades físicas, motores, perceptivas y cognitivas'. Medina (2017) destaca que la literatura es vasta cuando se trata de relaciones entre funciones cognitivas entre deportistas. A ese respecto, parece consolidado que los deportistas experts o de elite presentan mejores resultados en pruebas cognitivas, cuando comparados y deportistas noveles (Huijgen et al., 2015; Lundgren, Högman, Näslund $\&$ Parling, 2016), siendo posible pensar que esta diferencia tiene influencia de la cantidad de entrenamiento.

Según McAuliffe (2004), durante los entrenamientos y las competiciones ocurren adaptaciones y aprendizaje de los procesos de atención y de raciocínio, lo que lleva a los deportistas a mejorar su capacidad de discriminación de los estímulos irrelevantes y falsos (fintas), optimizando la eficiencia y sus tiempos de respuesta, diferenciando los noveles de los experientes, basicamente en las habilidades específicas de cada deporte. Alves y Araújo (1996) y Moran (2012) afirman que, cuanto más complejas son las situaciones, más se perciben las diferencias cognitivas de los deportistas con más experiencia, con relación a los noveles, principalmente el conocimiento táctico y la toma de decisiones. Según Williams et al., (2005), diversas investigaciones han tratado de estudiar cómo se forman los deportistas expertos en los deportes, analizando las capacidades perceptivas, cognitivas generales y específicas, como forma de atender a los procesos de desarrollo deportivo y a sus características cognitivas. También han sido estudiadas las posibles correlaciones entre la inteligencia general, la específica y el rendimiento deportivo en varios contextos, principalmente en los juegos deportivos colectivos (Matias \& Greco, 2010; Memmert, 2010).

Costa et al. (2002) realizaron un estudio para analizar las posibles correlaciones entre inteligencia y conocimiento específico de jugadores de fútbol con diferentes niveles competitivos. Fueron realizados las Pruebas de Barragen de Toulosse-Pieron (TP), Figuras Idénticas de Thurstone, Matrizes Progressivas de Raven (MP38) y cuestionarios específicos de conocimiento de fútbol, en jugadores de varios niveles de rendimiento. Los resultados indicaron diferencias significativas en el conocimiento específico de fútbol, obteniendo los jugadores con más experiencia mejores resultados que los noveles. Con relación a las pruebas cognitivas, las diferencias no fueron significativas, obteniendo los jugadores noveles resultados próximos a los obtenidos por los de más experiencia. Estos resultados muestran que ocurre una diferenciación mayor en las capacidades y en los conocimientos específicos del deporte en función de los entrenamientos y de las competiciones, lo que es corroborado por los estudios de Memmert (2010), Ozel, Larue, y Molinaro (2004) y Moreno, Moreno, Gil, García-González y Del Villar (2016).

Las principales diferencias entre deportistas noveles y expertos parecen ser más evidentes con relación a las habilidades cognitivas específicas de cada deporte, indicando que las adaptaciones y los aprendizajes se dan de forma más consistente en los procesos cognitivos específicos de cada situación deportiva. Para Konzag (1990), MacMahon y McPherson (2009), McPherson (1999), y Moran (2004), los deportistas con un nivel elevado de 
rendimiento tienen un conocimiento más elaborado, estructurado, organizado y sofisticado que los noveles con relación a las situaciones de juego.

En un metaanálisis, Voss, Kramer, Basak, Prakash y Roberts (2009) analizaron 128 estudios sobre la relación de las habilidades cognitivas básicas y específicas con el nivel de habilidades deportivas. Se constato que los estudios, en su mayoría, encontraron diferencias significativas entre deportistas expertos y noveles. Pero, al profundizar en el análisis de los datos y repasar los resultados de varios estudios y métodos, las diferencias no fueron estadísticamente significativas. También hubo divergencia entre las habilidades evaluadas, encontrándose diferencias más acentuadas en las habilidades más específicas (velocidad de procesamiento y raciocínio visoespacial), y menores en las habilidades básicas (habilidades atencionales básicas). Un factor considerado por los autores como limitación de los estudios fue la ausencia de control del tiempo de entrenamiento y de vivencias de los deportistas, ya que este factor influye en la amplitud del aprendizaje cognitivo.

Para Memmert (2010), al analizar los estudios se puede comprobar que muchos evaluaron a deportistas universitarios, lo que puede enmascarar los resultados, ya que los deportistas universitarios no pasan por procesos de entrenamiento y por competiciones con un volumen e intensidad suficiente como para producir modificaciones sustanciales en sus habilidades cognitivas, lo que puede llevar a conclusiones equivocadas. Según Voss et al. (2009), otro punto crítico en relación con la evaluación de las habilidades cognitivas en el deporte es la dificultad de correlacionar los resultados de las evaluaciones formales con el rendimiento deportivo, pues muchos de los instrumentos y test psicológicos no son suficientemente sensibles para diferenciar las habilidades específicas de los deportistas, lo que dificulta los procesos de evaluación.

Específicamente refiriéndose a Brasil, diferentemente del contexto de otros países, principalmente estadounidenses y europeos, el proceso de evaluación en el contexto deportivo presenta deficiencias y limitaciones en función de la poca disponibilidad de instrumentos específicos y aplicados a los deportes, y también limitaciones en términos de evaluación del contexto específico. Angelo y Rubio (2011) destacan que muchas veces se aplican pruebas y cuestionarios de otra área sin una validación y adaptación al contexto. Otras veces las pruebas aplicadas no están fundamentadas en las teorías psicológicas, lo que dificulta el processo de evaluación en psicologia del deporte. A ese respecto, es importate destacar que la aplicación de cuestionarios y pruebas deberá siempre ser precedida de processos de validación en el contexto deportivo, pues si esto no ocurre, los resultados obtenidos en las evaluaciones muchas veces no tienen relación con el rendimiento y con las necesidades del contexto deportivo y de los profesionales implicados en los entrenamientos y competiciones (Angelo \& Rubio, 2011).

Por las consideraciones hechas, y teniendo en cuenta la importancia de pruebas psicólogicas con propiedades psicométricas específicas para el contexto del deporte, los objetivos del presente estudio son: (1) evaluar las habilidades cognitivas de atención y raciocínio visoespacial en jugadores de voleibol de alto nível, comparando los resultados con la muestra normativa de los manuales de las pruebas, que fueron compuestas por indivíduos con las mismas edades, pero sin la práctica deportiva; (2) comparar el nivel de habilidades cognitivas en diferentes categorías/edades en voleibol, entendiendo que pueden existir posibles diferencias, pues pese a todos ser jugadores de elite, el tiempo de entrenamiento (años totales y tiempo semanal) es diferente entre las categorías, y; (3) conocer los índices y magnitudes de las correlaciones entre las habilidades cognitivas y el rendimiento deportivo en competición, por medio del índice general de rendimiento más utilizado en Brasil. Esto tal vez proporcionará evidencia de validez a las pruebas para el contexto deportivo de voleibol de alto rendimiento. 


\section{Método}

\section{Participantes}

Participaron en el estudio 159 jugadores de voleibol de alto nivel, pertenecientes a las selecciones brasileñas infantil (16), infanto (29), juvenil (28) y adulto (32) que disputaron los campeonatos 2013, y también adultos de equipos profesionales (54) que participaron en la Super Liga Nacional Brasileña de 2012/13, con tiempo medio de entrenamiento de 8.21 años $(D E=4.11)$. En relación al sexo, fueron $49(31 \%)$ mujeres y $110(69 \%)$ hombres. La edad osciló entre los 16 y 44 años $(M=21.60 ; D E=5.13)$, formándose subgrupos para el estudio de acuerdo con las categorias deportivas, es decir, infantil 16 años, infanto17/18 años $(M=17.45$; $D E=0.45)$, juvenil $19 / 20$ años $(M=19.31 ; D E=0.47)$ y adulto por encima de 21 años $(M=25.80 ; D E=5.54)$. El nivel de escolaridad varió entre la enseñanza media y superior.

\section{Instrumentos}

Bateria Psicológica para Evaluación de la Atención - BPA (Rueda, 2013). La BPA evalúa la capacidad general de atención y los tipos específicos de Atención Concentrada (AC), Atención Dividida (AD) y Atención Alternada (AA). El tiempo de aplicación de cada prueba varía de acuerdo con las instrucciones del manual. Son considerados en la corrección los aciertos, los errores y las omisiones, siendo el resultado final el total de aciertos menos los errores y las omisiones. El tiempo de aplicación es de 2 minutos, 4 minutos y 2 minutos y 30 segundos para la evaluación de la atención concentrada, dividida y alternada, respectivamente. La fiabilidad fue calculada por el método test-retest para los tres tipos de atención y en diferentes grupos de edades, con un intervalo entre siete y 14 dias. Los coeficientes variaron de .79 a .81. Con relación a las evidencias de validez, fue analizada por la relación con otras variables, como la edad y escolaridad, y por validez convergente, con las pruebas de atención concentrada (TEACO-FF), dividida (TEADI) y alternada (TEALT).

Escala de Atención Selectiva Visual - EASV (Sisto \& Castro, 2011). La EASV evalúa la capacidad del individuo para seleccionar estímulos visuales, identificando regularidades entre el modelo presentado y los estímulos disponibles para la selección. El test consiste en la presentación de cinco figuras con tres, seis o nueve formas geométricas dentro de cada una. La primera es una figura modelo, y en las otras cuatro deberá ser observada cual de ellas tiene más formas geométricas iguales a la figura modelo. Deberá ser marcada la opción que presente más coincidencias de formas geométricas con la figura modelo. El tiempo de aplicación es de 9 minutos y son considerados sólo los aciertos. La fiabilidad fue calculada utilizando el alfa de Cronbach (.88) y el modelo de Rasch (.90). Las evidencias de validez fueron verificadas por el proceso de respuesta y el nivel de dificultad de los ítems, así como por un estudio de estructura interna.

Test de los Cubos: para la evaluación del raciocínio visoespacial (Rueda \& Muniz, 2012). Evalúa el raciocínio visoespacial y está compuesto por 15 problemas que son resueltos mediante la observación del dibujo de un cubo de Rubik en la parte superior de la hoja, que debe ser comparado con los tres cubos presentados posteriormente abajo en la hoja, donde se encuentran instrucciones con el número de rotaciones que deben ser imaginadas en el cubo. Deberá marcarse qué figura del cubo corresponde a la alternativa correcta, en función de la orientación de cada hoja. El tiempo de aplicación es de 30 minutos. La fiabilidad fue evaluada por el alfa de Cronbach y del método Spearman-Brown, verificando que 13 de los 15 problemas presentaron coeficientes superiores a .70. La validez convergente fue analizada por la relación con el Subtest de Raciocínio Espacial de la Batería de Pruebas de Raciocínio, obteniendo un coeficiente de 61 . 
Data Volley (Data Project, 2010). El programa de "scout" evalúa de forma informatizada, las acciones deportivas en voleibol, generando puntuaciones técnicas y ranking de los jugadores, y ha sido desarrollado por la empresa italiana DataProject.com. Se trata de un software frecuentemente empleado en estudios sobre análisis del juego en voleibol (De la Vega-Marcos, Ruiz-Barquín, Tejero-González \& Rivera-Rodríguez, 2014; Drikos, Kountouris, Laios \& Laios, 2009; Marelic, Resetar \& Jankovic, 2004; Silva, Lacerda \& Joâo, 2013). El programa evalúa el resultado de la ejecución de los fundamentos del voleibol (saque, recepción, colocación, ataque, defensa y bloqueo), separadamente, considerando las acciones acertadas, las erradas y su nivel de eficacia. Cada uno de estos fundamentos es codificado en índices, variando de menos dos (-2) a dos (2) puntos, generando las puntuaciones técnicas de cada jugador, por set o juego. La puntuación menos dos (-2) indica error absoluto, menos uno (-1) indica error relativo, cero (0) indica un acierto sin eficacia, uno (1) indica un acierto con eficacia moderada, y dos (2) indica una eficacia absoluta. Los jugadores son evaluados por la ejecución de fundamentos específicos de acuerdo con la función que ejercen en su participación en el juego. De este modo, los colocadores son evaluados en saque, en colocación, en bloqueo y en defensa. Los jugadores de punta son evaluados en saque, en ataque, en bloqueo, en recepción y en defensa. Los jugadores centrales son evaluados por su saque, ataque, bloqueo y defensa, y los líberos, sólo por la recepción y por la defensa.

Las evaluaciones de las acciones son realizadas por dos experts en dicho programa, que son entrenados por la Confederación Brasileña de Voleibol (CBV), siendo evaluados en la precisión de sus resultados, periódicamente. Los datos obtenidos por los dos observadores durante los juegos son almacenados en el programa, generando una puntuación única que es la media entre los dos observadores expertos. Las puntuaciones son codificadas y descritas por fundamentos de juego y agrupadas en un índice general, obtenido de la media general de los fundamentos con relación a la función específica de cada jugador. De esta manera, se genera un índice general de rendimiento (IGR) para cada jugador, que varía de 0 a 100 , indicando el nivel técnico del jugador en cada set o juego, y en el campeonato. En este artículo fue utilizado el IGR correspondiente a uno de los campeonatos sudamericanos.

\section{Procedimiento}

La aplicación de las pruebas fue realizada siguiendo las explicaciones contenidas en los manuales y las normas específicas de aplicación. La aplicación fue realizada en la siguiente secuencia: primero las pruebas de atención (BPA y EASV), y posteriormente el Test de los Cubos, procurando tener un período de cinco minutos entre las pruebas. La aplicación ocurrió en el periodo de una semana antes de los campeonatos, y fue realizada en salas aisladas, con orientaciones específicas y normalizadas. La evaluación del rendimiento deportivo fue realizada por medio del análisis de los datos obtenidos por el programa de scout Data Volley (IGR), relativo a cada jugador en el campeonato Sudamericano de la categoría.

\section{Análisis de los datos}

Para tratar los datos se realizó un análisis descriptivo de los resultados de las pruebas y se comparó con los resultados de los manuales, con el padron de los testes psicológicos. Seguidamente, se realizo un análisis de varianza (ANOVA) en función de las variables de las categorías deportivas, y la prueba de Tukey para verificar las diferencias entre los grupos. Para analizar las correlaciones entre las medidas y el rendimiento deportivo se utilizó la correlación de Pearson. 


\section{Resultados}

Las puntuaciones obtenidas en las pruebas pueden observarse en la Tabla 1. En la BPA, los jugadores alcanzaron la puntuación máxima (120) en todos los test. Los mejores resultados fueron obtenidos en Atención Alternada, en el cual 34 obtuvieron la nota máxima. En el EASV ningún jugador consiguió obtener la nota máxima (30). En el Test de los Cubos, sólo un jugador obtuvo la nota máxima (15).

Tabla 1

Estadística descriptiva de las pruebas de Atención y Raciocínio Visoespacial de jugadores y de los manuales $(N=159)$.

\begin{tabular}{clrrrrrr} 
& \multicolumn{7}{c}{ BPA } \\
\cline { 2 - 8 } & Jugadores & AC & AD & AA & $\begin{array}{r}\text { Atención } \\
\text { General }\end{array}$ & EASV & Cubos \\
\cline { 2 - 8 } & Min & 10 & 44 & 46 & 116 & 8 & 2 \\
& Max & 120 & 120 & 120 & 360 & 28 & 15 \\
Jugadores & $M$ & 92.09 & 91.08 & 105.44 & 289.32 & 17.13 & 9.09 \\
& $D E$ & 18.89 & 18.19 & 15.20 & 42.79 & 3.59 & 2.28 \\
\hline Datos de los & $M$ & 82.85 & 64.66 & 82.89 & 230.44 & 15.76 & 8.24 \\
Manuales & $D E$ & 26.80 & 33.47 & 30.29 & 79.61 & 5.02 & 2.89 \\
& $T$ & 6.57 & 21.1 & 20.98 & 19.46 & 5.38 & 6.74 \\
& $P$ & $<.001$ & $<.001$ & $<.001$ & $<.001$ & $<0.001$ & $<.001$ \\
\hline
\end{tabular}

Nota: AC atención concentrada; $\mathrm{AD}$ atención dividida; AA atención alternada

Al comparar las medias de las pruebas con la de los manuales se observa que los jugadores obtuvieron medias superiores en todas las medidas, presentando diferencias estadísticamente significativas $(p<.001)$, y también presentaron resultados más homogéneos, con desviaciones típicas menores. De forma general, se puede verificar que los jugadores presentaron cierta facilidad en la BPA. Las notas mínimas fueron superiores a los manuales y también un porcentaje mayor de jugadores (8\%) obtuvo notas máximas. En la EASV y Cubos las medias obtenidas por los jugadores fueron también superiores a las de los manuales, además, presentaron resultados más próximos, indicando un grado de dificultad mayor.

Seguidamente, se realizó un análisis de datos basado en las categorías deportivas, de acuerdo con las franjas de edad establecidas en voleibol. Primero, se realizó un análisis de varianza (ANOVA) de las medidas de las pruebas con las categorías deportivas. Los datos se observan en la Tabla 2.

Tabla 2

Medidas de las pruebas de Atención y de Raciocínio Visoespacial en función de las categorías deportivas

\begin{tabular}{lcc}
\hline & $F$ & $P$ \\
\hline Atención Concentrada & 2.678 & .048 \\
Atención Dividida & 2.697 & .047 \\
Atención Alternada & 2.679 & .048 \\
Atención General & 3.171 & .025 \\
Atención Selectiva Visual & 0.707 & .549 \\
Test de los Cubos & 0.691 & .558 \\
\hline
\end{tabular}


Al observar los datos, se puede verificar que la BPA presentó diferencias estadísticamente significativas $(p<.05)$ entre las categorías deportivas. Hay que destacar, sin embargo, que las diferencias presentadas tienen pequeña magnitud (AC, AD, AA) (Dancey \& Reidy, 2006). En las pruebas de atención EASV y de raciocínio visoespacial (Cubos), no se encontraron diferencias estadísticamente significativas. Posteriormente, se realizó la prueba de Tukey para verificar el resultado de qué categorias podrían explicar esas diferencias (Tabla $3)$.

Tabla 3

Prueba de Tukey en las pruebas de Atención Dividida y Total.

\begin{tabular}{lccccc}
\hline Categoría deportiva & $n$ & \multicolumn{2}{c}{ Atención Dividida } & \multicolumn{2}{c}{ Atención General } \\
\cline { 2 - 6 } & & 1 & 2 & 1 & 2 \\
\cline { 2 - 6 } Infantil & 17 & 82.29 & & 271.82 & \\
Juvenil & 40 & 89.98 & 89.98 & 283.98 & 283.98 \\
Adulto & 97 & 92.08 & 92.08 & 294.88 & 294.88 \\
Infanto & 46 & & 96.30 & & 302.22 \\
$P$ & & 0.11 & 0.46 & 0.11 & 0.28 \\
\hline
\end{tabular}

Se observó que sólo la categoría infantil e infanto fueron diferenciadas con relación a las demás categorías. Posteriormente, para verificar las posibles relaciones entre las pruebas de atención y el raciocínio visoespacial con el rendimiento deportivo de los jugadores, se realizó una correlación de Pearson para la muestra total y para cada una de las categorías deportivas. Esos datos pueden observarse en la Tabla 4.

Tabla 4

Coeficientes de correlación entre los resultados de la BPA, EASV, Test de los Cubos y o rendimiento deportivo para la muestra total y categorías deportivas.

\begin{tabular}{|c|c|c|c|c|c|c|c|c|}
\hline \multirow{9}{*}{ 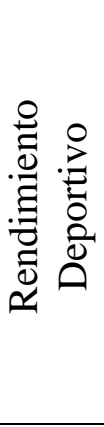 } & \multirow{3}{*}{\multicolumn{2}{|c|}{ Categoría }} & \multicolumn{3}{|c|}{ BPA } & & & \\
\hline & & & $\mathrm{AC}$ & $\mathrm{AD}$ & AA & General & EASV & Cubos \\
\hline & & & $R$ & $R$ & $R$ & $r$ & $R$ & $R$ \\
\hline & \multicolumn{8}{|c|}{$n$} \\
\hline & General & 159 & .11 & 0.09 & .02 & .10 & -0.02 & .10 \\
\hline & Infantil & 16 & .11 & -0.34 & -.46 & -.23 & -0.08 & -.31 \\
\hline & Infanto & 29 & -.03 & 0.17 & .15 & .10 & 0.23 & $.53 * *$ \\
\hline & Juvenil & 28 & .31 & $0.40^{*}$ & $.41 *$ & $.44^{*}$ & 0.32 & .09 \\
\hline & Adulto & 86 & .08 & 0.05 & -.04 & .05 & -0.14 & -.07 \\
\hline
\end{tabular}

$* p<.05-* * p<.001$

Al observar los datos en la Tabla 4 se puede verificar que los resultados de los test de atención BPA en relación a la muestra general y a las categorías infantil, infanto y adulto, no presentaron correlaciones estadísticamente significativas $(p<.05)$ entre los test y el rendimiento deportivo. En la categoría juvenil, los resultados indicaron correlaciones estadísticamente significativas con relación a las pruebas de atención dividida, alternada y general, presentando magnitud baja (Dancey \& Reidy, 2006).

En cuanto al test de atención selectiva visual, no se encontraron correlaciones estadísticamente significativas en ninguna categoría. En el Test de los Cubos sólo se encontraron correlaciones estadísticamente significativas en la categoría infanto $(p<.001)$, 
con magnitud moderada, y en las demás categorías las correlaciones no fueron consideradas estadísticamente significativas.

\section{Discusión}

Uno de los objetivos de este estudio fue analizar los factores de atención y de raciocínio visoespacial en jugadores de voleibol de alto nivel, comparando los datos con las medias de la muestra normativa de los manuales de los test, en los cuales son presentados datos de la muestra de la población brasileña. Los resultados obtenidos por los jugadores presentan medias superiores en todas las pruebas (BPA, EASV y Cubos), con diferencias significativas con relación a las medias normativas de los manuales, lo que viene a corroborar los resultados de estudos anteriores (Afonso et al., 2012; Alves et al., 2013; Gorman, Abernethy \& Farrow, 2013) demostrando la superioridad en las habilidades cognitivas evaluadas en los jugadores, en relación a la población de forma general. De esta forma, los resultados observados confirman el desarrollo de los jugadores, tanto en la capacidad de atención (BPA, EASV) como en raciocínio visoespacial (Cubos), no sólo en niveles mayores que la media de la población, sino también de forma más homogénea, presentando menor desviación típica en todas las pruebas. Así, se puede concluir que los jugadores de alto rendimiento de voleibol presentan niveles de atención general, selectiva visual y de raciocínio visoespacial más desarrollados que las medias correspondientes a las muestras normativas de los manuales de cada prueba en todas las categorías deportivas comparando com as respectivas idades. Este resultado permite afirar y corroborar las afirmaciones de que deportistas de elite presentan desempeños superiores en pruebas cognitivas que deportistas noveles o no deportistas (Huijgen et al., 2015; Lundgren et al., 2016). Sin embargo, el tiempo de entrenamiento no parece ser una variable que halla influenciado los desempeños en las pruebas.

Se realizó también un análisis para verificar si había diferencias entre los resultados de las pruebas con relación a las categorías deportivas (en este artículo entendidos como experts y noveles), encontrándose solamente en las que componen la BPA. De las diferencias encontradas, solamente entre las categorías infantil e infanto pueden ser consideradas estadísticamente significativas. Esa diferencia entre las categorías infantil e infanto ocurre probablemente en función de la diferencia de tiempo de entrenamiento realizado por las categorías, siendo un año para la infantil, y 2 a 3 años para la infanto (Castro et al., 2016). De acuerdo con Rueda (2013), los resultados a partir de los 20 años presentan una reducción en los índices de los test, lo que probablemente influye en los resultados de las categorías juvenil y adulto, que son mayores que esta edad. Estos resultados sugieren que el mismo sea utilizado solamente en las categorías de base (infantil/infanto) visto que no detecta, de forma clara, la evolución de las variables en relación a las categorías más avanzadas (juvenil/adulto).

Con relación a EASV y al Test de los Cubos, los resultados del análisis por categorías no presentaron diferencias significativas, demostrando que los mismos no consiguen diferenciar la evolución de las habilidades entre las categorías. Estos valores probablemente se dan en función de la dificultad de las pruebas y del alto nivel de los jugadores. También debe considerarse que, según los datos de los manuales de los test (Rueda \& Muniz, 2012; Sisto \& Castro, 2011), después de los 20 años, las habilidades de atención selectiva visual y de raciocínio visoespacial comienzan a presentar una caída en sus resultados que se van acentuando con la edad. Como en la categoría juvenil los jugadores tienen entre 20 y 21 años, y en la categoría adulto ellos están por encima de 22 años, los resultados parecen confirmar las tendencias propuestas por los manuales, aproximando los datos de las categorías anteriores. Se verificó también que, entre la categoría juvenil y la categoría adulto, hubo una reducción en las medias, apreciándose que, esta caída fue más acentuada en los índices de los 
manuales que entre los resultados de los jugadores, en todas las pruebas. Lo que, según los estudios de Memmert (2010) y Memmert et al. (2009), puede ser entendido que, en función de la estimulación por la participación en los entrenamientos y en las competiciones, por los jugadores de alto rendimiento, ocurre un cierto mantenimiento del rendimiento de esas habilidades.

Al correlacionar los resultados con el rendimiento deportivo, se verificó que, en la muestra general, no se encontraron correlaciones significativas entre ninguna de las pruebas y el rendimiento deportivo, medido mediante el programa Data Volley. Estos resultados se deben, en parte, a la pequeña diferencia encontrada entre las categorías en las pruebas de atención y de raciocínio visoespacial. También tenemos que considerar las características del instrumento utilizado para evaluar el rendimiento deportivo (Data Volley), que analiza el rendimiento de los jugadores en situación de juego, siempre considerando a los jugadores de forma comparativa con relación a jugadores del mismo nivel. Los resultados del programa de evaluación ofrecen datos significativos a los entrenadores con relación al rendimiento de los jugadores en los juegos, pero no diferencian con relación a una evolución entre las diferentes categorías de juego.

Al analizar las correlaciones de acuerdo con las categorías de juego, sólo se encontraron correlaciones significativas en la categoría juvenil en las medidas de atención dividida, alternada y general de la BPA. Estos resultados indican que cuando se considera la categoría deportiva, el rendimiento deportivo evaluado por el programa Data Volley, correlaciona con la puntuación obtenida en la BPA, solamente en la franja de edad entre 20 y 21 años.

En el Test de los Cubos, según Rueda y Muniz (2012), ocurre la evolución del raciocínio visoespacial hasta los 19 años, y después de esa edad se inicia una caída de los índices. En función de esto, en la categoría infanto todavía se presentan correlaciones con el rendimiento deportivo, sabiendo que en esa categoría se tiene una edad entre 17 y 18 años. En la EASV no se encontraron correlaciones en ninguna categoría de juego.

Concluyendo, se puede afirmar que los jugadores tienen mejores resultados que las muestras de los manuales (Rueda \& Muniz 2012) en todas las habilidades cognitivas evaluadas. Los resultados, cuando son analizados por categorías deportivas, presentan los mismos comportamientos de la población de forma general, diferenciándose solamente en la categoría adulto, en la cual presenta una caída menos significativa en los índices en función de los entrenamientos y de las competiciones.

Los resultados de las pruebas cognitivas no presentan correlaciones significativas con relación a los datos de rendimiento deportivo, índice general de rendimiento (IGR), obtenidos por el programa Data Volley, pues mediante él, se evalúa el rendimiento por categorías, comparando los resultados de los jugadores con relación a equipos del mismo nível, no distinguiendo los jugadores de categorías diferentes. Probablemente, como los jugadores son todos de alto rendimiento en su categoría, presentan resultados muy próximos, que no los diferencian en cuanto a rendimiento deportivo. Los valores obtenidos por los jugadores mediante el Data Volley dependen del rival con el que se enfrentan, ya que se obtienen por la actuación de los jugadores ante un rival determinado. Esto puede hacer que, en distintas categorías de juego, los jugadores tengan niveles de rendimiento similares, pero siempre comparados con la exigencia de su propia competición, y con relación a los rivales con los que se han enfrentado. Pero, los instrumentos de evaluación utilizados deberían también ser aplicados a jugadores de niveles diferentes de rendimiento, procurando identificar las características de los jugadores de iniciación deportiva y de categorías intermedias, lo que los haría más adaptados al contexto deportivo de forma general. Hay que mencionar que el índice utilizado no distingue los fundamentos específicos y permite apenas un IGR. Estudios que 
analicen los fundamentos de forma separada, ciertamente podrán contribuir para profundizar los hallazgos de esta investigación.

De esa forma, se observa que las pruebas de atención (BPA y EASV) aplicados presentan pequeña correlación con el rendimiento deportivo, lo que los hace limitados para los procesos de evaluación y para seguir el desarrollo de jugadores de alto rendimiento. El test de los Cubos puede ser aplicado en las categorías más desarrolladas como un instrumento auxiliar en la evaluación y seguimiento de los jugadores, ya que se ha visto que presenta resultados más evidentes de desarrollo de la habilidad evaluada.

Se sugiere que se realicen nuevos estudios utilizando las pruebas (BPA, EASV y Cubos), en deportistas de categorías iniciales de formación deportiva, y también que se apliquen otros instrumentos de rendimiento deportivo en los estudios con categorías de alto rendimiento, con la intención de buscar correlaciones entre las diversas fases de desarrollo deportivo y las habilidades cognitivas. También se sugiere que las pruebas de habilidades cognitivas basados en situaciones deportivas cotidianas y aplicados mediante ordenadores, puedan ser utilizados por los psicólogos y entrenadores como factor de diagnóstico en la evaluación de diversas fases del entrenamiento en voleibol. Se recomienda también que otros instrumentos sean estudiados y validados en el contexto del voleibol de alto nivel, ya que se ha visto que los procesos de evaluación y de seguimiento de las habilidades cognitivas no deben restringirse sólo a la aplicación de test. Igualmente, se sugiere que se apliquen instrumentos de evaluación de forma más dinámica (vídeos) y contextualizada con el voleibol de alto rendimiento (Arroyo, 2012; Castro, Costa, Praça, Campbel \& Grego, 2017), y que los test sean aplicados a otras modalidades deportivas, buscando validarlos para el contexto deportivo.

\section{Referencias}

Abernethy, B. (2001). Attention. En R. Singer, A. H. Hausenblas \& C. M. Janelle (Eds.). Handbook of sport psychology (pp. 53-85). New York: Wiley.

Afonso, J., Garganta, J., McRobert, A., Williams, A.M., \& Mesquita, I. (2012). The perceptual cognitive processes underpinning skilled performance in volleyball: Evidence from eye-movements and verbal reports of thinking involving an in situ representative task. Journal of Sports Science and Medicine, 11, 339-345.

Alves, H., Voss, M. W., Boot, W. R., Deslandes, A., Cossich, V., Salles, J. I., y Kramer, A. F. (2013). Perceptual-Cognitive expertise in elite Volleyball player. Frontier in Psychology, 4(36), 1-9. doi: 10.3389/fpsyg.2013.00036

Alves, J., \& Araújo, D. (1996). Processamento de informações e tomada de decisão no esporte. En J. F. Cruz (Ed). Manual de psicologia do desporto (pp. 361-388). Braga: SHO Ltda.

Angelo, L. F., \& Rubio, K. (2011). Instrumentos de avaliação em psicologia doesporte. São Paulo: Casa do Psicólogo.

Arroyo, J. S. D. (2012). Análisis de las estrategias de búsqueda visual y la respuesta de reacción en jugadores de voleibol ante una situación de recepción del saque. (Disertación doctoral). Universidad de Extremadura).

Beilock, S. L., Carr, T. H., MacMahon, C., \& Starkes, J. L. (2002). When paying attention becomes counter productive: Impact of divided versus skill-focused attention on novice and experienced performance of sensorimotor skills. Journal of Experimental psychology applied, 8(1), 6-16. doi: 10 137//1076.898X8.1.6

Cárdenas, D., Perales, J. C., \& Alarcón, F. (2015). La planificación del entrenamiento para la toma de decisiones en los deportes de equipo. El entrenamiento táctico y decisional en el deporte. Madrid: Síntesis. 
Carroll, J.B. (1993). Human cognitive abilities: A survey of factor-Analytic Studies. UK: Cambridge University Press.

Castaneda, B., \& Gray, R. (2007). Effects of focus of attention on baseball batting performance in players of differing skill levels. Journal of Sport \& Exercise Psychology, 29, 60-77.

Castro, H, O., Praça, G., De Conti, G. \& Greco, P.J. (2016). Visual behavior and the quality of decision-making on volleybol. Revista Brasileira de Cineantropomentria \& desempenho humano, 18, 638-647.

Castro, H.O., Costa, G. D. C. T., Praça, G. M., Campbell, C. S. G., \& Greco, P. J. (2017). Análise das fixações visuais e tomada de decisão de atletas de voleibol das categorias infanto e juvenil. Revista Brasileira de Ciência e Movimento, 25(1), 51-59.

Conejero, M.S, Serenini, A.L.P, Fernández-Echeverria, C., Collado-Mateo, D. \& Moreno, M.P. (2020). The effect of decision Training, from a cognitive perspective, on decision-Making in Volleybol: A systematic reviw and meta-analysis. International Journal Environmente Research and Public Health, 17. doi:10.3390/ijerph17103628.

Conejero, M.S, Serenini, A.L.P, Gonzáles-Silva, J, \& Moreno, M.P. (2020). Factor used to make appropriate decisions in youth categories in Volleiball. Sustainability, 12. doi: $10.3390 /$ su 12145633

Costa, J, C., Garganta, J., Fonseca, A., \& Botelho, M. (2002). Inteligência e conhecimento específico em jovens futebolistas de diferentes níveis competitivos. Revista Portuguesa Ciência Desporto, 2(4), 7-20.

Dancey, C., \& Reidy, J. (2006). Estatística sem matemática para psicologia: usando SPSS para windows. Porto Alegre: Artmed.

DataProject.com - Sport software - (2010) programa de computador (versão 3). Recuperado de http//www.dataproject.com.

De la Vega-Marcos, R., Ruiz-Barquín, R., Tejero-González, C., \& Rivera-Rodríguez, M. (2014). Relación entre estados de ánimo y rendimiento em voleibol masculino de alto nível. Revista de Psicología del Deporte, 23(1), 49-56.

Dick, M., Button, C., \& Davids, K. (2010). Examination of gaze behaviours under in situ and video simulation task constraints reveals differences in information pickup for perception and action. Attention, Perception, \& Psychophysics, 72(3), 706-720. doi: 10.3758/APP.72.3.706

Drikos, S., Kountouris, P., Laios, A. \& Laios, Y. (2009). Correlates of team performance in volleyball. International Journal of Performance Analysis of Sport, 9(2), 149-156. doi: $10.1080 / 24748668.2009 .11868472$

Emanuel M., Jarus, T., \& Bart, O. (2008). Effect of focus of attention and age on motor acquisition, retention, and transfer: a randomized trial. Physical Therapy, 88(2), 251260. doi: $10.2522 / \mathrm{ptj} .20060174$

García, J. S., Garcés, E. F. R., \& Jara, P. V. (2005). El papel de la atención en el ámbito deportivo: una aproximación bibliométrica de la literatura recogida en la base de datos Psyclit. Revista de Psicología del Deporte, 14(1), 125-140.

Gorman, A. D.; Abernethy, B., \& Farrow, D. (2013). The expert advantage in dynamic pattern recall persists across both attended and unattended display element. Attention \& Percept Psychophys, 75, 835-844. doi:10.3758/s13414-013-0423-3

Gray, R. (2004). Attending to the execution of a complex sensorimotor skill: Expertise differences, choking, and slumps. Journal of Experimental Psychology Applied, 10(1), 42-54. doi: 10.1037/1076-898X.10.1.42 
Huijgen, B., Leemhuis, S., Kok, N. M., Verburgh, L., Oosterlaan, J., ElferinkGemser, M. T., $\&$ Visscher, C. (2015). Cognitive functions in elite and sub-elite youth soccer players aged 13 to 17 years. PLoS ONE, 10(12). doi: 10.1371/journal.pone.0144580

Janelle, C., \& Hillman, C. (2003). Expert Performance in Sport: Current perspectives and critical issues. En J. L. Starkes \& K. A. Ericsson (Eds.), Expert Performance in Sports: Advances in Research on Sport Expertise (pp. 19-47). Champaign, IL: Human Kinetics.

Konzag, I. (1990). Attivita cognitiva e formazione del giocatore. Revista di Cultura Sportiva, 20, 14-20.

Lundgren, T., Högman, L., Näslund, M., \& Parling, T. (2016). Preliminary investigation of executive functions in elite ice hockey players. Journal of Clinical Sport Psychology, 10(4), 324-335. doi: 10.1123/jcsp.2015-0030.

MacMahon, C. \& McPherson, S. L. (2009). Knowledge base as a mechanism for perceptualcognitive tasks: skills is in the details! International Journal Sport Psychology 40, 565579.

Marelic, N., Resetar, T., \& Jankovic, V. (2004). Discriminant analysis of the sets won and the sets lost by one team in A1 italian volleyball league - a case study. Kinesiology, 36(1), $75-82$.

Matias, C., \& Greco, P. J. (2010). Cognição \& ação nos jogos esportivos coletivos. Ciência \& Cognição, 15(1), 252-271.

McAuliffe, J. (2004). Differences in attentional set between athletes and nonathletes. The journal of General Psychology, 13(4), 426-427. doi: 10.3200/GENP.131.4.426-437

McGrew, K. (2009). CHC theory and the human cognitive abilities project: standing on the shoulders of the giants of psychometric intelligence research. Intelligence, 37, 1-10. doi: 10.1016/j.intell.2008.08.004

McPherson, S. L. (1999). Expert-novice differences in performance skills and problem representations of youth and adults during tennis competition. Research Quarterly for Exercise and Sport, 70, 233-251. doi: 10.1080/02701367.1999.10608043

Medina, J. A. (2017). Incidencia del tipo de actividad física en las funciones ejecutivas en jóvenes deportistas (Disertación doctoral). Universidad Católica San Antonio de Murcia). doi::10.13140/RG.2.2.34032.35844

Medina-Cascales, J. A., Alarcón-López, F., Castillo-Díaz, A., \& Cárdenas-Vélez, D. (2019). Efecto del ejercicio y la actividad física sobre las funciones ejecutivas en niños y en jóvenes. Una revisión sistemática. SPORT TK-Revista EuroAmericana de Ciencias del Deporte, 8(2), 43-53

Memmert, D. (2010). Creativity, expertise, and attention: exploring their development and their relationships. Journal of Sport \& Exercise Psychology, 3, 1-10. doi: 10.1007/s 12662-012-0237-9

Memmert, D., Simons, D. J., \& Grimme, T. (2009). The relationship between visual attention and expertise in sports. Psychology of Sport and Exercise, 10, 146-151. doi: 10.1016/j.psychsport.2008.06.002

Miçooğulları, O. B., \& Kirazci, S. (2012). Effects of Internal, External and Preference of Attentional Focus Feedback Instructions on Learning Soccer "Head Kick". Monten Journal Sports Science Medicine, 1(1). 21-26.

Moran, A. P. (2004). Sport and exercise Psychology - A critical introduction, New York: Routledge.

Moran, A. P. (2012). Thinking in action: some insights from cognitive sport psychology. Thinking Skills and Creativity, 7, 85-92. doi: 10.1010/j.tsc.2012.03.005 
Moreno, M. P., Moreno, A., Gil-Arias, A., García-González, L., \& Del Villar, F. (2016). Influence of experience in procedural knowledge in volleyball: Applications for training. International Journal of Sports Science \& Coaching, 11(2), 191-199. doi: $10.1177 / 1747954116637152$

Orlick, T. (2007). In pursuit of excellence: How to win in sport and life through mental training. Champaing, IL: Human Kinects.

Ozel, S., Larue, J., \& Molinaro, L. (2004). Relation between sport and spatial imagery: Comparison of three groups of participants. The Journal of Psychology, 138(1), 4963. doi:10.1007/s10459-006-9021-7

Ripoll, H. (1991). Analysis of visual scanning patterns of volleyball players in problem solving task. International Journal of Sport Psychology, 19(9), 9-25.

Rueda, F. J. M. (2013). Bateria Psicológica de Avaliação da Atenção - BPA. (Relatório não publicado, Universidade São Francisco, Itatiba, SP, Brasil).

Rueda, F. J. M., \& Muniz, M. (2012). Testes dos Cubos para avaliação do raciocínio visuoespacial. São Paulo: Vetor.

Sánchez-López, J. Fernández, T., Silva-Pereyra, J., Martínez-Mesa, J. A., \& Moreno-Aguirre, A. J. (2014). Evaluación de la atención en deportistas de artes marciales. Expertos vs. Novatos. Revista de Psicología del Deporte, 23(1), 87-94.

Schneider, J., \& McGrew, K. (2012). The Cattell-Horn-Carroll (CHC) model of Intelligence $v 2,2$ : Theoretical perspective, a visual tour and summary (online). Retrieved from http//www,iapsych,com,/IAPWEB/CHCTheory,html.

Silva, M., Lacerda, D., \& Joâo, P.V. (2013). Match analysis of discrimination skills according to the setter attack zone position in high level volleyball. International Journal of Performance Analysis in Sport, 13(2), 452-460. doi: 10.1080/24748668.2013.11868661

Sindik, J.; Botica, A. \& Fiškuš, M. (2015). Preliminary psychometric validation of the Multidimensional inventory of sport excellence: attention scales and mental energy. Monten Journal Sports Science Medicine, 4(2), 17-28.

Sisto, F. F., \& Castro, N. R. (2011). Escala de Atenção Seletiva Visual- EASV. São Paulo: Casa do Psicólogo.

Sternberg, R, J. (2000). Psicologia cognitiva. Porto Alegre: Artmed.

Suárez-Cadenas, E., Courel-Ibáñez, J., \& Cárdenas-Vélez, D. (2017). La toma de decisiones en baloncesto. Una propuesta de árboles decisionales para la enseñanza del bloqueo directo. Acción Psicológica, 14(1), 43-56.

Swann, C., Moran, A., \& Piggot, D. (2015). Defining elite athletes: Issues in the study of expert performance in psychology. Psychology of Sport and Exercise, 16, 3-14. doi: 10.1016/j.p.2014.07.004

Thomas, K. T. (1994). The development of sport expertise: From leeds to MVP. Legend Quest, 46, 211-222. doi: 10.1080/00336297.1994.10484121

Voss, M.W., Kramer, A. E., Basak, C., Prakash, R.S., \& Roberts, B. (2009). Are expert athletes "experts" in the cognitive laboratory? A meta analytic review of basic attention and perception and sport expertise. Applied Cognition Psychology, 24, 812-826. doi: 10.1016/j.bandc.2014.03.007

Williams, A. M., Davids, K., \& Williams, J. G. (2005). Visual perception and action in sport. London: Taylors e Francis e-Book.

Wulf, G., \& Prinz, W. (2001). Directing attention to movement effects enhance learning: a review. Psychonomic Bulletin \& Review, 8(4), 648-660. 
Wulf, G., Shea, C. H., \& Park, J. (2001). Attention and motor performance: Preferences and advantages of external focus. Research Quarterly for Exercise and Sport, 72(4), 335344. doi: 10.1080/02701367.2001.10608970

Wulf, G., \& Su, J. (2007). An external focus of attention enhances golf shot accuracy in beginner's experts. Research Quarterly for Exercise and Sport, 78(4). doi: 10.1080/02701367.2007.10599436

Este artículo es resultado de la tesis doctoral del primer autor, bajo la orientación y coorientación del segundo y tercer autor, respectivamente.

Financiamiento: Esta investigación obtuvo apoyo financiero de la Coordenação de Aperfeiçoamento de Pessoal de Nível Superior de Brasil - CAPES.

Contribución de los autores: a) Concepción y diseño del trabajo; b) Adquisición de datos; c) Análisis e interpretación de datos; d) Redacción del manuscrito; e) revisión crítica del manuscrito.

A.L.P.S ha contribuido en a,b,c,d,e; F.J.M.R en a,c,d,e; M.P.M. en c,e.

Editora científica responsable: Dra. Cecilia Cracco 